\title{
Endovascular Treatment of Ruptured Dissecting Aneurysms on the P2-3 Segment of the Posterior Cerebral Artery: A Report of Two Cases
}

\author{
Seonah Choi ${ }^{1}$, Joonho Chung ${ }^{1,2}$ \\ ${ }^{1}$ Department of Neurosurgery, Severance Hospital, Yonsei University College of Medicine, Seoul, Korea \\ ${ }^{2}$ Severance Institute for Vascular and Metabolic Research, Yonsei University College of Medicine, Seoul, Korea
}

Received: July 16, 2019

Accepted: July 31, 2019

Corresponding Author: Joonho Chung, M.D., Ph.D. Department of Neurosurgery, Stroke Center, Severance Hospital Yonsei University College of Medicine 50-1, Yonsei-ro, Seodaemun-gu, Seoul, 03722, Korea

Tel: +82-2-2228-2150

Fax: +82-2-393-9979

E-mail: ns.joonho.chung@gmail.

com

\begin{abstract}
Intracranial ruptured dissecting aneurysms can have fatal outcomes. Among them, posterior cerebral artery dissecting aneurysm with hemorrhage is uncommon but usually fatal due to its deep location and proximity to surrounding critical structures. Most previously published reports on this type of aneurysm have concluded that the neurologic outcome can be improved with the use of certain surgical or endovascular approaches. However, in severe cases with a poor initial clinical status, patient improvement should not be expected. We report two cases of P2-3 segment ruptured dissecting aneurysms that were treated by external ventricular drainage for control of increased intracranial pressure followed by one-stage endovascular treatment for the aneurysm.
\end{abstract}

Keywords: Dissecting aneurysm; Endovascular treatment; Parent artery occlusion; Posterior cerebral artery

\section{INTRODUCTION}

Posterior cerebral artery (PCA) dissecting aneurysm is rare and typically presents with ischemic symptoms in the PCA territory and subarachnoid hemorrhage ${ }^{10,15)}$. Once aneurysmal rupture occurs along with subarachnoid hemorrhage, a poor outcome can be expected such as a high modified Rankin Scale (mRS) score or a low overall survival rate; therefore, immediate and proper treatment is necessary. Due to aneurysmal location and associated crucial anatomy, initial neurological status is unfavorable in most of these patients. In these cases, endovascular treatment (EVT) is usually adopted for fast intervention of the aneurysm's natural course. EVT includes simple coiling, balloon remodeling, stent-assisted coiling or using flow diverter devices as a reconstruction technique, and deconstruction techniques, such as parent artery occlusion. In the case of a ruptured dissecting aneurysm, either deconstruction or reconstruction is feasible, and their strengths and weaknesses are well known among neurosurgeons and neurointerventionists. As a result, the optimal approach for each case may vary according to anatomical and morphological features of each aneurysm. However, devastating results (mRS 3-6) are very likely despite the best treatments. We report 2 cases of P2-3 segment dissecting aneurysms that were treated with external ventricular drainage (EVD) for control of increased intracranial pressure (ICP) followed by EVT but still experienced morbidity and mortality.

Copyright (C) 2019 The Korean Neurointensive Care Society

This is an Open Access article distributed under the terms of the Creative Commons Attribution Non-Commercial License (http://creativecommons.org/licenses/by-nc/4.0/) which permits unrestricted non-commercial use, distribution, and reproduction in any medium, provided the original work is properly cited. 


\section{CASE REPORTS}

\section{Case 1}

A 50-year-old female patient with no previous medical problems presented to the emergency department with deep stupor mental status and was admitted. Her initial Hunt-Hess grade was 4. Her computed tomography (CT) scan revealed intraventricular hemorrhage and intraparenchymal hemorrhage (Fig. 1A). CT an- giography showed a wide neck right P2 aneurysm suspicious for dissection. The patient underwent EVD prior to digital subtraction angiography (DSA) due to acute hydrocephalus and poor Hunt-Hess grade. DSA revealed a right PCA dissecting aneurysm (Fig. 1B and C), and one-stage stent-assisted coil embolization (Enterprise stent $4.5 \mathrm{~mm} \times 28 \mathrm{~mm}$ ) was carried out. The aneurysm was completely obliterated, and the PCA was patent (Fig. 1D). The patient remained vegetative (modified Rankin Scale 5)
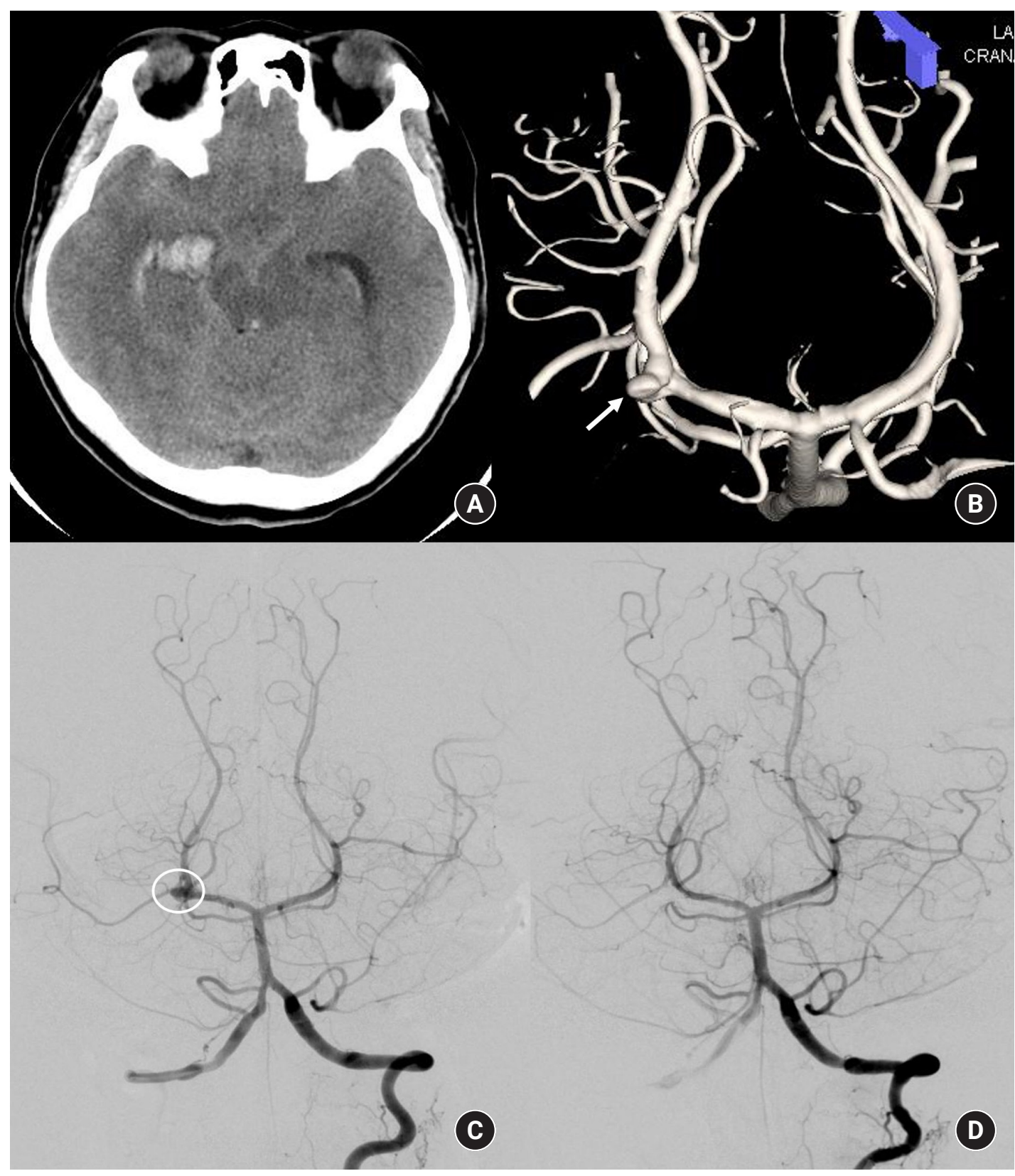

Fig. 1. A 50-year-old female with an initial Hunt-Hess grade 4. (A) Computed tomography obtained in the emergency department shows intraventricular, intraparenchymal and subarachnoid hemorrhages. (B) A right P2 segment dissecting aneurysm was noted on threedimensional reconstruction image and (C) on digital subtraction angiography. (D) The aneurysm was completely occluded by stent-assisted coil embolization. 
at the 24-month clinical follow-up, and a repeat DSA was not performed.

\section{Case 2}

A 64-year-old female patient presented to the emergency department with semicomatous mental status and pupil dilatation. Her CT scan revealed a large amount of basal ganglia and thala- mus intraparenchymal hemorrhage combined with intraventricular and subarachnoid hemorrhage and increased basal cistern attenuation (Fig. 2A). Her initial Hunt-Hess grade was 5, and her modified Fisher grade was 4 . We performed EVD and catheterization for intraparenchymal hemorrhage. DSA showed a left P2-3 dissecting aneurysm greater than $20 \mathrm{~mm}$ in size, and a distal PCA was noted at the end of the aneurysm (Fig. 2B). Parent artery oc-
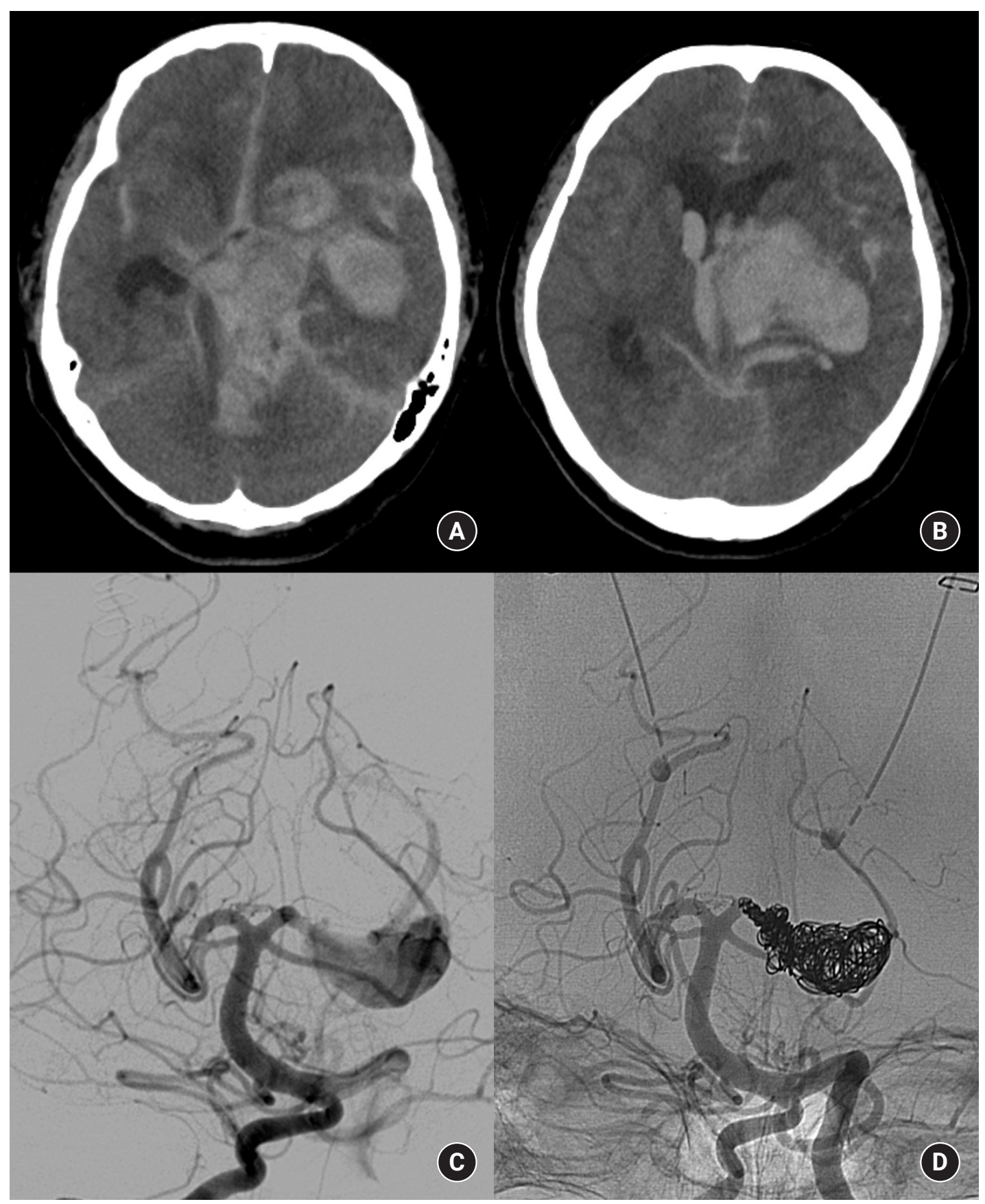

Fig. 2. A 64-year-old female with a initial Hunt-Hess grade 5. (A) Computed tomography reveals a large amount of intraparenchymal, intraventricular and subarachnoid hemorrhage, including basal cisterns. (B) Digital subtraction angiography reveals a left P2-3 segment dissecting aneurysm; a distal PCA was noted at the end of the aneurysm. (C) Parent artery occlusion was performed immediately by coil embolization. 
clusion was performed immediately by coil embolization (Fig. 2C). However, the patient died 10 days after the procedure due to increased ICP and brainstem dysfunction.

\section{DISCUSSION}

PCA aneurysms are uncommon, with an incidence $<1 \%$. Most PCA aneurysms are non-saccular type ${ }^{10)}$. More than 80 $90 \%$ of all intracranial dissections occur in the posterior circulation, but almost all are located near the vertebral and basilar arteries and have a male predominance ${ }^{13,15)}$. In contrast, PCA dissection is extremely rare, occurs at a younger age, and has a female predominance; only a few cases have been reported ${ }^{10,15)}$. Among these reported cases, only a few ruptured P2-3 dissecting aneurysms are documented. The suggested causes of this type of dissection include syphilis, migraine, cystic medial necrosis, Marfan syndrome, mixed connective tissue disease, fibromuscular dysplasia, homocystinuria, polycystic kidney disease, and trauma ${ }^{15,16)}$. However, our two cases had no previous associated medical histories or medications.

The most common presentation is subarachnoid hemorrhage with headache and neurological deficits that defer depending on the involved segments of the PCA ${ }^{2,10,16)}$. In general, PCA aneurysms arise mostly on $\mathrm{P} 1$ or at the $\mathrm{P} 1-2$ junction $^{8,9)}$, but dissecting aneurysms usually involve P2 or P2-3 segments ${ }^{10,16)}$; therefore, associated neurologic deficits include memory impairment, oculomotor palsy, visual disturbance (homonymous hemianopsia) and sometimes hemiparesis due to mass effect ${ }^{2}$. Acute hydrocephalus should be a concern when presented because it aggravates mental deterioration. Our cases, both suffered mental status deterioration, so focal neurologic deficits could not be examined. The natural history of dissecting aneurysm is not yet well described but

Table 1. List of P2-3 dissecting aneurysm ruptured including the present case

\begin{tabular}{|c|c|c|c|c|c|c|}
\hline Authors & Age & Sex & Intial HHG & PCA segments & Treatment & Outcomes (mRS) \\
\hline Berger and Wilson (1984) [1] & 21 & $\mathrm{~F}$ & 2 & $\mathrm{P} 2$ & Surgical clipping(vessel occlusion) & 2 \\
\hline \multirow{3}{*}{ Lanzinski et al. (2000) [9] } & 48 & $\mathrm{~F}$ & 3 & P2-3 & Parent artery occlusion & 1 \\
\hline & 17 & $\mathrm{M}$ & 4 & P2-3 & Parent artery occlusion & 0 \\
\hline & 13 & $\mathrm{~F}$ & 4 & $\mathrm{P} 2$ & Parent artery occlusion & 0 \\
\hline Kitazawa et al. (2001) [7] & 43 & $\mathrm{M}$ & 1 & $\mathrm{P} 2$ & surgical clipping (vessel occlusion) & not mentioned \\
\hline \multirow[t]{4}{*}{ Hallacq et al. (2002) [5] } & 20 & $\mathrm{M}$ & 2 & $\mathrm{P} 2$ & Coil embolization & 0 \\
\hline & 60 & $\mathrm{M}$ & 2 & $\mathrm{P} 2$ & Coil embolization & 0 \\
\hline & 49 & $\mathrm{~F}$ & 3 & $\mathrm{P} 2$ & Coil embolization & 1 \\
\hline & 47 & $\mathrm{M}$ & 2 & $\mathrm{P} 2$ & Coil embolization & 1 \\
\hline Hamada et al. (2005) [6] & 33 & $\mathrm{~F}$ & 4 & $\mathrm{P} 2$ & Surgical clipping (vessel occlusion) & 0 \\
\hline \multirow[t]{8}{*}{ Lv et al. (2009) [12] } & 37 & $\mathrm{~F}$ & 4 & $\mathrm{P} 2$ & Parent artery occlusion & 0 \\
\hline & 49 & M & 4 & $\mathrm{P} 2$ & Parent artery occlusion & 0 \\
\hline & 43 & $\mathrm{M}$ & 4 & $\mathrm{P} 2$ & Parent artery occlusion & 0 \\
\hline & 48 & $\mathrm{M}$ & 3 & $\mathrm{P} 2$ & Parent artery occlusion & 0 \\
\hline & 58 & $\mathrm{~F}$ & 4 & $\mathrm{P} 2$ & Parent artery occlusion & 1 \\
\hline & 31 & $\mathrm{M}$ & 2 & $\mathrm{P} 2$ & Parent artery occlusion & 0 \\
\hline & 4 & $\mathrm{M}$ & 2 & $\mathrm{P} 2$ & Parent artery occlusion & 0 \\
\hline & 5 & $\mathrm{M}$ & 2 & $\mathrm{P} 2$ & Parent artery occlusion & 0 \\
\hline \multirow[t]{6}{*}{ Taqi et al. (2011) [16] } & 23 & $\mathrm{~F}$ & 4 & $\mathrm{P} 2$ & Stent-assisted coiling & not mentioned \\
\hline & 48 & $\mathrm{~F}$ & 2 & $\mathrm{P} 2$ & Stent-assisted coiling & not mentioned \\
\hline & 5 & $\mathrm{~F}$ & 2 & P2-3 & Parent artery occlusion & not mentioned \\
\hline & 45 & $\mathrm{~F}$ & 4 & $\mathrm{P} 2$ & Parent artery occlusion & not mentioned \\
\hline & 25 & M & 4 & P3 & Parent artery occlusion & not mentioned \\
\hline & 54 & $\mathrm{~F}$ & 2 & $\mathrm{P} 2-3$ & Stent-assisted coiling & not mentioned \\
\hline Krishnan et al. (2014) [8] & 36 & $\mathrm{~F}$ & 4 & $\mathrm{P} 2$ & Surgical clipping (vessel occlusion) & 2 \\
\hline Osman et al. (2017) [14] & 49 & $\mathrm{~F}$ & 2 & $\mathrm{P} 2$ & Stent-assisted coiling & 0 \\
\hline Cunegatto-Braga et al. (2018) [3] & 4 & M & 2 & $\mathrm{P} 2$ & Flow diverter device & 0 \\
\hline \multirow{3}{*}{$\begin{array}{l}\text { Choi and Chung } \\
\text { (the present cases) }\end{array}$} & 50 & $\mathrm{~F}$ & 4 & P2-3 & Stent-assisted coiling & 5 \\
\hline & & & & & & \\
\hline & 64 & $\mathrm{~F}$ & 5 & $\mathrm{P} 2-3$ & Parent artery occlusion & 6 \\
\hline
\end{tabular}


notorious for poor outcome of $30-70 \%$ re-bleeding risk if not treated $^{13)}$.

Diagnosis of dissecting aneurysm is usually made based upon DSA findings of "pearl and string (a fusiform dilatation associated with proximal or distal narrowing)," "double lumen (visualization of two channels)," and "fusiform dilatation" ${ }^{\text {"1,16) }}$. CT angiography may be an alternative option, but it may not reveal an aneurysm if a mass effect exists, as we encountered with Case 2. Magnetic resonance angiography can also demonstrate the location and length of the dissection, but it has drawbacks of motion artifacts and limitations due to the small caliber of the vessels in this area ${ }^{15)}$. Therefore, DSA is the gold standard.

Due to its hard-to-access location, typical presence of numerous perforators, and limitations in clipping dissecting aneurysms4), endovascular approaches have been attempted for PCA aneurysm treatment. The primary goal is isolation of the aneurysms and preservation of the parent artery ${ }^{10}$. If the situation allows, simple coiling, balloon remodeling, and stent-assisted coiling may also be performed to achieve the goal ${ }^{10)}$. However, parent artery occlusion is inevitable despite the risk of PCA territory infarction. Usually, occlusion of the P2 segment or the distal segment of the PCA is tolerable due to purulent collateral circulations; if not, a combined distal bypass could be a rescue treat$m e n t^{10}$. For the last several years, flow diverters have been used in various vessels even smaller than the PCA ${ }^{3,11)}$, and this could be an option for dissecting PCA aneurysms. however, the required accompanying anticoagulant/antiplatelet therapy could worsen the patient's situation, so treatment decisions should involve great care $^{3,10,13,15)}$.

Previous articles have shown that, with proper management, almost all P2-3 segment dissecting aneurysm cases had favorable clinical outcomes (Table 1$)^{1,3,-9,12,14,16)}$. The difference of the present cases from those previously published is clinical outcome (mRS 5 and 6) despite complete occlusion of the aneurysm during the acute period. Considering that isolated PCA dissections are known to present with ischemic symptoms and still have favorable outcomes ${ }^{15}$, our cases could be classified as unusual among PCA dissections. The patients' initial clinical status (such as Hunt-Hess grade), amount of hemorrhage, and presence of brainstem injuries likely had a greater impact than other factors on their clinical outcomes. Unfortunately, we were unable to achieve good outcomes in our cases, but we believe and recommend that a combined surgical and endovascular treatment approach should be considered first to control both increased ICP and future re-bleeding.

\section{CONCLUSION}

Patients with ruptured P2-3 segment dissecting aneurysms might be expected to experience unfavorable outcomes due to the aneurysm's deep location within surrounding critical structures. However, EVD for ICP control followed by one-stage EVT for the aneurysm could be a safe and feasible treatment option.

\section{NOTES}

\section{Conflict of interest}

we declare that we have no conflict of interest.

\section{Ethical approval}

all procedures performed in the studies involving human participants were in accordance with the ethical standards of our Institutional Review Board with the 1964 Helsinki Declaration and its later amendments or comparable ethical standards.

\section{Informed consent}

in this retrospective study, the requirement for informed consent was waived.

\section{ACIKNOWLEDGEMENTS}

No funding was received for this study.

\section{REFERENCES}

1. Berger MS, Wilson $\mathrm{CB}$. Intracranial dissecting aneurysms of the posterior circulation. Report of six cases and review of the literature. J Neurosurg 1984;61:882-894.

2. Ciceri EF, Klucznik RP, Grossman RG, Rose JE, Mawad ME. Aneurysms of the posterior cerebral artery: classification and endovascular treatment. AJNR Am J Neuroradiol 2001;22:27-34.

3. Cunegatto-Braga M, Hogan B, Aguilar-Salinas P, Bejer AD, Hanel RA. Pipeline embolization device flow diversion for a dissecting ruptured posterior cerebral artery aneurysm in a pediatric patient. World Neurosurg 2018;117:255-260.

4. Goehre F, Jahromi BR, Lehecka M, Lehto H, Kivisaari R, Andrade-Barazarte $\mathrm{H}$, et al. Posterior Cerebral Artery Aneurysms: Treatment and Outcome Analysis in 121 Patients. World Neurosurg 2016;92:521-532.

5. Hallacq P, Piotin M, Moret J. Endovascular occlusion of the posterior cerebral artery for the treatment of $\mathrm{P} 2$ segment aneurysms: retrospective review of a 10-year series. AJNR Am J Neuroradiol 2002;23:1128-1136. 
6. Hamada J, Morioka M, Yano S, Todaka T, Kai Y, Kuratsu J. Clinical features of aneurysms of the posterior cerebral artery: a 15year experience with 21 cases. Neurosurgery 2005;56:662-670.

7. Kitazawa K, Tanaka Y, Muraoka S, Okudera H, Orz Y, Kyoshima $\mathrm{K}$, et al. Specific characteristics and management strategies of cerebral artery aneurysms: report of eleven cases. J Clin Neurosci 2001;8:23-26.

8. Krishnan P, Kartikueyan R, Thamatapu E, Chowdhury SR. Dissecting aneurysm of the posterior cerebral artery: Defining the role of deliberate surgical proximal artery occlusion. J Neurosci Rural Pract 2014;5:443-445.

9. Lazinski D, Willinsky RA, TerBrugge K, Montanera W. Dissecting aneurysms of the posterior cerebral artery: angioarchitecture and review of the literature. Neuroradiology 2000;42:128-133.

10. Liu L, He H, Jiang C, Lv X, Li Y. Deliberate parent artery occlusion for non-saccular posterior cerebral artery aneurysms. Interv Neuroradiol 20111;17:159-168.

11. Lozupone E, Piano M, Valvassori L, Quilici L, Pero G, Visconti $\mathrm{E}$, et al. Flow diverter devices in ruptured intracranial aneurysms: a single-center experience. J Neurosurg 2018;128:1037-
1043.

12. Lv X, Li Y, Jiang C, Yang X, Wu Z. Parent vessel occlusion for P2 dissecting aneurysms of the posterior cerebral artery. Surg Neurol 2009;71:319-325.

13. Oran I, Cinar C, Yağci B, Tarhan S, Kiroğlu Y, Serter S. Ruptured dissecting aneurysms arising from non-vertebral arteries of the posterior circulation: endovascular treatment perspective. Diagn Interv Radiol 2009;15:159-165.

14. Osman MW, Kadziolka K, Peirot L. Optional endovascular therapy of dissecting posterior cerebral artery aneurysm. Interven Neurol 2017;6:219-228.

15. Sherman P, Oka M, Aldrich E, Jordan L. Isolated posterior cerebral artery dissection: Report of three cases. AJNR Am J Neuroradiol 2006;27:648-652.

16. Taqi MA, Lazzaro MA, Pandya DJ, Badruddin A, Zaidat OO. Dissecting aneurysms of posterior cerebral artery: clinical presentation, angiographic findings, treatment, and outcome. Front Neurol 2011;2:38. 